\title{
Numerical investigation of minimum drag profiles in laminar flow using deep learning surrogates
}

\author{
Li-Wei Chen, Berkay Alp Cakal, Xiangyu Hu, Nils Thuerey
}

September 2020

\begin{abstract}
Efficiently predicting the flowfield and load in aerodynamic shape optimisation remains a highly challenging and relevant task. Deep learning methods have been of particular interest for such problems, due to their success for solving inverse problems in other fields. In the present study, U-net based deep neural network (DNN) models are trained with highfidelity datasets to infer flowfields, and then employed as surrogate models to carry out the shape optimisation problem, i.e. to find a drag minimal profile with a fixed cross-section area subjected to a two-dimensional steady laminar flow. A level-set method as well as Bézier-curve method are used to parameterise the shape, while trained neural networks in conjunction with automatic differentiation are utilized to calculate the gradient flow in the optimisation framework. The optimised shapes and drag force values calculated from the flowfields predicted by DNN models agree well with reference data obtained via a Navier-Stokes solver and from the literature, which demonstrates that the DNN models are capable of predicting not only flowfield but also yield satisfactory aerodynamic forces. This is particularly promising as the DNNs were not specifically trained to infer aerodynamic forces. In conjunction with the fast runtime, the DNN-based optimisation framework shows promise for general aerodynamic design problems.
\end{abstract}

\section{Introduction}

Owing to the importance in a wide range of fundamental studies and industrial applications, a significant effort has been made to study the shape optimisation for minimising aerodynamic drag over a bluff body [1, 2]. The deployment of computational fluid dynamics tools has played an important role in these optimisation problems [3]. While a direct optimisation via high-fidelity computational fluid dynamics (CFD) models gives reliable results, the high computational cost of each simulation, e.g., for Reynolds-averaged Navier-Stokes formulations, and the large amount of evaluations needed, lead to assessments that such optimisations are still not feasible for the practical engineering [4]. When considering gradient-based optimisation, the adjoint method provides an effective way to 
calculate the gradients of an objective function w.r.t. design variables and alleviates the computational workload greatly 5,9$]$, but the number of required adjoint CFD simulations is typically still prohibitively expensive when multiple optimisation objectives are considered [10]. In gradient-free methods (e.g. genetic algorithm), the computational cost rises dramatically as the number of design variables is increased, especially when the convergence requirement is tightened [11]. Therefore, advances in terms of surrogate-based optimisation are of central importance for both gradient-based and gradient-free optimisation methods [12, 13].

Recently, state-of-the-art deep learning methods and architectures have been successfully developed to achieve fast prediction of fluid physics. Among others, Bhatnagar et al. [14] developed convolutional neural network (CNN) method for aerodynamics flowfields, while others studied the predictability of laminar flows 15], employed graph neural networks to predict transonic flows 16], or learned reductions of numerical errors in PDE fluid solvers [17]. For the inference of Reynolds-averaged Navier-Stokes (RANS) solutions, a U-net based deep learning model was proposed and shown to be significantly faster than a conventional CFD solver [18]. These promising achievements open up new possibilities of applying DNN-based flow solvers in the aerodynamic shape optimisation. In the present study we focus on evaluating the accuracy and performance of DNN-base surrogates in laminar flow regimes.

Modern deep learning methods are also giving new impetus to aerodynamic shape optimisation research. E.g., Eismann et al. [19] used a data-driven Bayesian approach to design optimization and generated object shapes with an improved drag coefficient. Viquerat and Hachem [20], evaluated quantitative predictions such as drag forces using a VGG-like convolutional neural network. To improve the surrogate-based optimisation, Li et al. 21] proposed a new sampling method for airfoils and wings based on a generative adversarial network (GAN). Renganathan et al. 222] designed a surrogate-based framework by training a deep neural network (DNN) that is used for gradient-based and gradientfree optimisations. In these studies, the neural network is mainly trained to construct the mapping between shape parameters and the aerodynamic quantities (e.g. lift and drag coefficients), but no flowfield information can be obtained from the network models. We instead demonstrate how deep learning models that were not specifically trained to infer the parameters to be minimized, can be used in optimisation problems. The proposed deep learning model offers two advantages. First, the model is flexible as it predicts a full flowfield in a region of interest. Once trained, it can be used in different optimization tasks with multiple objectives. This is of particular importance when considering problems such as compressor/turbine blade wake mixing [23]. Second, as the model is differentiable, it can be seamlessly integrated with deep learning algorithms [24, 25].

To understand the mechanisms underlying drag reduction and to develop optimisation algorithms, analytical and computational work have been specifically performed for Stokes' flow and laminar steady flow over a body [26 32]. As far back as 1970s, Pironneau [26] analysed the minimum drag shape for a given 
volume in Stokes flow, and later for the Navier-Stokes equations [27]. By using the adjoint variable approach, Kim and Kim [30] investigated the minimal drag profile for a fixed cross-section area in the two-dimensional laminar flow with the Reynolds number range of $R e=1$ to 40 . More recently Katamine et al. 31] studied the same problem at two Reynolds numbers $R e=0.1$ and $R e=40$. With theoretical and numerical approaches, Glowinski and Pironneau [28, 29] looked for the axisymmetric profile of given area and smallest drag in a uniform incompressible laminar viscous flow at Reynolds numbers between 100 and $10^{5}$ and obtained a drag-minimal shape with a wedge of angle $90^{\circ}$ at the front end and a cusp rear end from an initial slender profile. Although the laminar flow regimes are well studied, due to the separation and nonlinear nature of the fluid, it can be challenging for surrogate models to predict the drag-minimal shape as well as aerodynamic forces. Moreover, with the Reynolds number approaching zero, the flowfield experiences a dramatic change from a separated vortical flow towards a creeping flow, which poses additional difficulties to the learning task. To our knowledge, no previous studies exist that target training a "generalised model" that performs well in such a Reynolds number range. We investigate this topic and quantitatively assess the results in the context of deep learning surrogates.

In the present paper, we adopt an approach for the U-net based flowfield inference [18] and use the trained deep neural network as flow solver in the shape optimisation. In comparison to conventional surrogate models [33] and other optimisation work involving deep learning [19 22], we make use of a generic model that infers flow solutions: in our case it produces fluid pressure and velocity as field quantities. I.e., given encoded boundary conditions and shape, the DNN surrogate produces a flowfield solution, from which the aerodynamic forces are calculated. Thus, both the flowfield and aerodynamic forces can be obtained during the optimisation. As we can fully control and generate arbitrary amounts of high-quality flow samples, we can train our models in a fully supervised manner. We use the trained DNN models in the shape optimisation to find the drag minimal profile in the two-dimensional steady laminar flow regime for a fixed cross-section area, and evaluate results w.r.t. shapes obtained using a full Navier-Stokes flow solver in the same optimisation framework. We specifically focus on the challenging Reynolds number range from 1 to 40. Both level-set and Bézier-curve based methods are employed for shape parameterisation. The implementation utilizes the automatic differentiation package of the PyTorch package [34], so the gradient flow driving the evolution of shapes can be directly calculated 35]. Here DNN-based surrogate models show particular promise as they allow for a seamless integration into the optimisation algorithms that are commonly used for training DNNs.

The purpose of the present work is to demonstrate the capability of deep learning techniques for robust and efficient shape optimisation, and for achieving an improved understanding of the inference of the fundamental phenomena involved in low Reynolds number flows. This paper is organized as follows. The mathematical formulation and numerical method are briefly presented in $\$ 2$ The neural network architecture and training procedure will be described in 


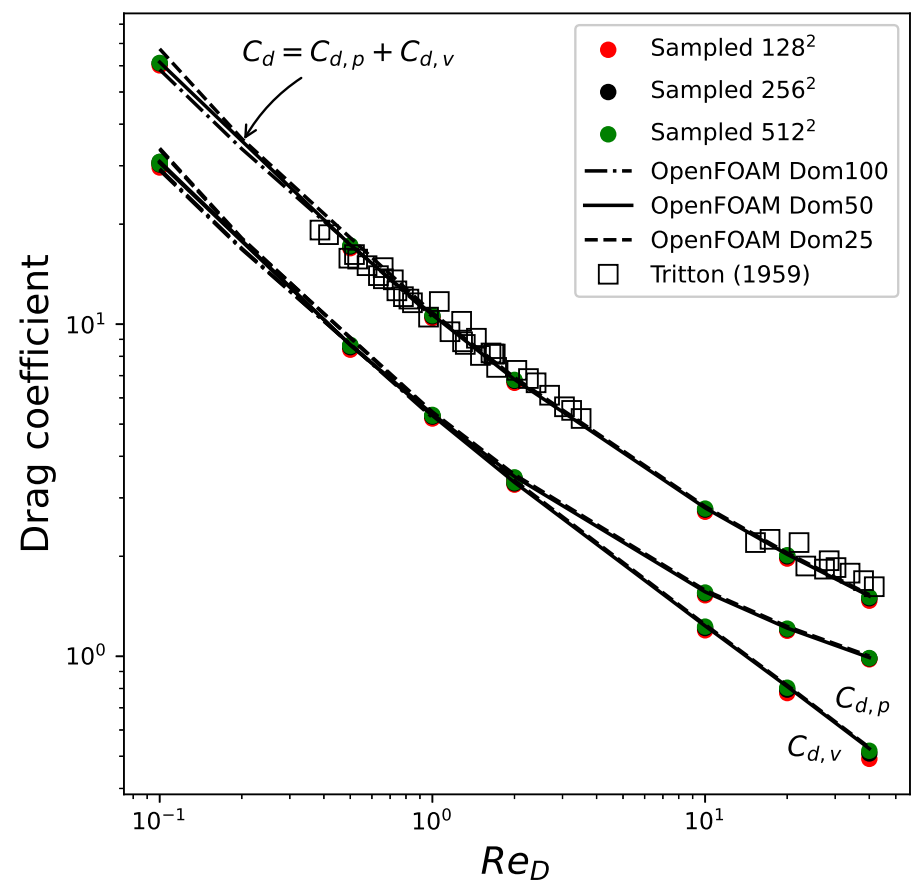

Figure 1: Drag coefficients from $R e_{D}=0.1$ to 40 . Surface integral values from OpenFOAM simulations are plot in black curves. Results based on re-sampled points on Cartesian grids with the resolution of $128 \times 128,256 \times 256$ and $512 \times 512$ are plot with red, black and green circles, respectively. All data are compared with the experimental measurements by Tritton (1959), which are shown by blue squares.

\$3. The detailed experiments and results are then given in $\$ 4$ and concluding remarks in $\$ 5$.

\section{Methodology}

We first explain and validate our approach for computing the fluid flow environment in which shapes should be optimised. Afterwards, we describe two different shape parameterisiations, a level-set and a Bézier curve based one, which we employ for our optimisation results. 


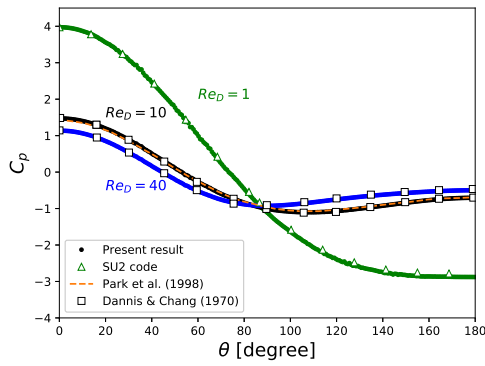

(a) Pressure coefficient distribution

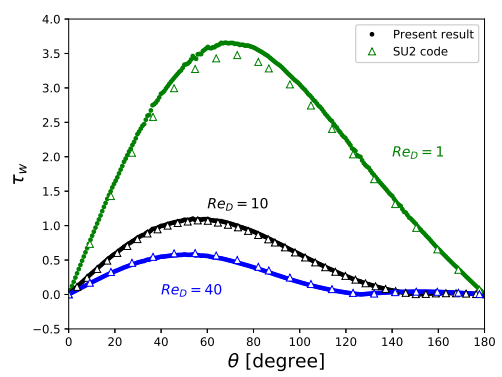

(b) Wall shear stress distribution

Figure 2: Pressure coefficient and wall shear stress distributions.

\section{$2.1 \quad$ Numerical procedure}

We consider two-dimensional incompressible steady laminar flows over profiles of given area and look for the minimal drag design. The profile is initialised with a circular cylinder and updated by utilizing steepest gradient descent as optimisation algorithm. The Reynolds number $R e_{D}$ in the present work is based on the diameter of the initial circular cylinder. It can be also interpreted that the the length scale is defined as the equivalent diameter for given area $S$ of an arbitrary shape, i.e. $D=2 \sqrt{S / \pi}$. In the present work, $D \approx 0.39424[\mathrm{~m}]$ is used.

To calculate the flowfield around the profile at each iteration of the optimisation, two methods are employed in the present study. The first approach is a conventional steady solver of Navier-Stokes equations, i.e. simpleFoam within the open-source package OpenFOAM (from https://openfoam.org/). The second one is the deep learning model [18], which is trained with flowfield datasets generated by simpleFoam that consists of several thousand profiles at a chosen range of Reynolds numbers. More details about the architecture of the neural network, data generation, training and performance will be discussed in $\$ 3$.

SimpleFoam is a steady-state solver for incompressible, turbulent flow using the Semi-Implicit Method for Pressure Linked Equations (known as "SIMPLE") [36]. The governing equations are numerically solved by a second-order finite volume method [37]. The unstructured mesh in the fluid domain is generated using open source code Gmsh version 4.4.1. To properly resolve the viscous flow, the mesh resolution is refined near the wall of the profile and the minimum mesh size is set as $\sim 6 \times 10^{-3} D$, where $D$ is the equivalent circular diameter of the profile. The outer boundary, where the freestream boundary condition is imposed, is set as $50[\mathrm{~m}](\sim 32 D)$ away from the wall (noted as "OpenFOAM DOM50"). The effects of domain size are assessed by performing additional simulations with domain size of $25[\mathrm{~m}]$ and $100[\mathrm{~m}]$ away from the wall (noted as "OpenFOAM DOM25" and "OpenFOAM DOM100", respectively). Here the drag coefficient $C_{d}$ is defined as the drag force divided by the projected length and dynamic head. As shown in figure 1 from $R e_{D}=0.1$ to $R e_{D}=40$, the 
total $C_{d}$ as well as the viscous $C_{d, v}$ and inviscid $C_{d, p}$ parts obtained from three different domains almost collapse. Although small differences when $\operatorname{Re}_{D}<0.5$ are observed, the predictions in the interested range $[1,40]$ are consistent and not sensitive to the domain size. The computation runs for 6000 iterations to obtain a converged state.

To validate the setup, we compare our numerical results and literature data in terms of the surface pressure coefficient and wall shear. As sanity checks for the numerical setup, we also run SU2 [see 38] with the same mesh for comparisons. Figure 2(a) shows the distribution of the surface pressure coefficient $\left(p_{w}-p_{\infty}\right) / 0.5 \rho_{\infty} U_{\infty}^{2}$ at $R e_{D}=1,10$ and 40 . Here, $\theta$ is defined as the angle of the intersection of the horizontal line and the vector of the center to a local surface point, so that $\theta=0^{\circ}$ is the stagnation point in the upwind side and $\theta=180^{\circ}$ in the downwind side. Only half of the surface distribution is shown due to symmetry. The results agree well with the numerical results by Dennis and Chang [39], and the results for OpenFOAM and SU2 collapse. In figure 2(b), the results for OpenFOAM compare well with the one predicted by SU2. The drag coefficients in the Reynolds numbers range from 0.1 to 40 agree well with the experimental data by Tritton et al. [40] in figure 1] which further supports that the current setup and the solver produce reliable data.

To facilitate neural networks with convolution layers, the velocity and pressure field from OpenFOAM in the region of interest are re-sampled with a uniform Cartesian grid in a rectangular domain $[-1,1]^{2}\left(\approx[-1.27 D, 1.27 D]^{2}\right)$. A typical resolution used in the present study is $128 \times 128$, corresponding to the grid size of $0.02 \mathrm{D}$. As also shown in figure 1 the effect of the resolution of re-sampling on the drag calculation has been studied. The details about the force calculation on Cartesian grids are given in 2.2 .1 Results with three different resolutions shown as colored symbols, i.e. $128^{2}, 256^{2}$, and $512^{2}$, compare favourably with the surface integral values based on the original mesh in OpenFOAM. Therefore, re-sampled fields on the $128 \times 128$ grid will be used in the deep learning framework and optimisation unless otherwise noted.

\subsection{Shape parameterisation}

\subsubsection{Level-set method}

The level set method proposed by Osher and Sethian [41] is a technique that tracks an interface implicitly and has been widely used in fluid physics, image segmentation, computer vision as well as shape optimisation [42 44]. The level set function $\phi$ is a higher dimensional auxiliary scalar function, the zero-level set contour of which is the implicit representation of a time-dependent surface $\Gamma(t)=\{\boldsymbol{x}: \phi(\boldsymbol{x})=0\}$. Here, let $\mathcal{D} \in \mathcal{R}^{\mathcal{N}}$ be a reference domain, $\boldsymbol{x} \in \mathcal{D}$ and $\Omega$ is a body created by the enclosed surface $\Gamma$. Specifically in the present study, the domain $\mathcal{D}$ is referred to the sampled Cartesian grid in the rectangular region, and $\mathcal{N}$ is 2 as we focus on two-dimensional problems. The level set function $\phi$ 
is defined by a signed distance function (SDF):

$$
\phi= \begin{cases}-d(\Gamma(t)) & x \in \Omega \\ 0 & x \in \partial \Omega(\text { on } \Gamma) \\ d(\Gamma(t)) & x \in \mathcal{D}-\Omega\end{cases}
$$

where $d(\Gamma(t))$ denotes the Euclidean distance from $\boldsymbol{x}$ to $\Gamma$.

The arc length $c$ and area $S$ of the body are formulated as $c=\int_{\mathcal{D}} \delta_{\epsilon}(\phi)|\nabla \phi| d s$ and $S=\int_{\mathcal{D}} H_{\epsilon}(-\phi) d s$. To make the operators differentiable, in the above, we use smoothed Heaviside and Dirac Delta function $H_{\epsilon}(x)=\frac{1}{1+e^{-x / \epsilon}}$ and $\delta_{\epsilon}(x)=\partial_{x} \frac{1}{1+e^{-x / \epsilon}}$, respectively. $\epsilon$ is a small positive number and chosen as twice of the grid size [45]. Then, the aerodynamic forces due to pressure distribution and viscous effect are described as

$$
\begin{gathered}
\boldsymbol{F}_{\text {pressure }}=\int_{\partial \Omega}(p \boldsymbol{n}) d l=\int_{\mathcal{D}}(p \boldsymbol{n}) \delta_{\epsilon}(\phi)|\nabla \phi| d s \\
\boldsymbol{F}_{\text {viscous }}=\int_{\partial \Omega}(\mu \boldsymbol{n} \times \boldsymbol{\omega}) d l=\int_{\mathcal{D}}(\mu \boldsymbol{n} \times \boldsymbol{\omega}) \delta_{\epsilon}(\phi)|\nabla \phi| d s .
\end{gathered}
$$

Here, $\boldsymbol{n}$ is the unit normal vector, $\boldsymbol{n}=\frac{\nabla \phi}{|\nabla \phi|}, p$ is the pressure, $\mu$ is the dynamic viscosity, and $\boldsymbol{\omega}=\nabla \times \boldsymbol{v}$ is the vorticity with $\boldsymbol{v}$ being the velocity. A nearest neighbour method is used to extrapolate values of pressure and vorticity inside the shape $\Omega$. Then, the drag force is considered as the loss in the optimisation, i.e.

$$
\mathcal{L}=\boldsymbol{F}_{\text {pressure }} \cdot \hat{\boldsymbol{i}}_{x}+\boldsymbol{F}_{\text {viscous }} \cdot \hat{\boldsymbol{i}}_{x}
$$

where $\hat{\boldsymbol{i}}_{x}$ is the unit vector in the direction of $x$ axis.

The minimisation of equation (44) is solved by the following equation:

$$
\frac{\partial \phi}{\partial \tau}+V_{n}|\nabla \phi|=0
$$

Here, the normal velocity is defined as $V_{n}=\frac{\partial \mathcal{L}}{\partial \phi}$. At every iteration, the Eikonal equation is solved numerically with the fast marching method to ensure $|\nabla \phi| \approx$ 1.0 [46]. Then, we have $\frac{\partial \phi}{\partial \tau} \propto-\frac{\partial \mathcal{L}}{\partial \phi}$, which is a gradient flow that minimises the loss function $\mathcal{L}$ and drives the evolution of the profile [4]. For a more rigorous mathematical analysis we refer to Kraft [35]. In the present work, the automatic differentiation fuctionality of PyTorch is utilized to efficiently minimize equation (4) via gradient descent. Note that the level-set based surface representation and optimisation algorithm are relatively independent modules, and can be coupled with any flow solver, such as OpenFOAM and SU2, so long as the solver provides a re-sampled flowfield on the Cartesian grid (e.g. $128 \times 128)$ at an iteration in the optimisation. We will leverage this flexibility by replacing the numerical solver with a surrogate model represented by a trained neural network below. 


\subsubsection{Bézier-curve based parameterisation}

Bézier curve based parametric shape parameterisation is a widely accepted technique in aerodynamic studies [48 50]. This work utilizes two Bézier curves, representing upper and lower surfaces of the profile denoted with superscript $k=\{u, l\}$. Control points $\mathbf{P}_{i}^{k} \in \mathcal{D}$ are the parameters of the optimisation framework. The Bézier curves are defined via following equation:

$$
\mathbf{B}^{k}(t)=\sum_{i=0}^{n}\left(\begin{array}{c}
n \\
i
\end{array}\right) t^{i}(1-t)^{n-i} \mathbf{P}_{i}^{k}
$$

where $t \in[0,1]$ denotes the sample points along the curves. First and the last control points of each curve share the same parameters to construct the closure $\Omega$ of the profile.

A binary labeling of the Cartesian grid $\mathcal{D}$ is performed as

$$
\chi= \begin{cases}1 & x \in \Omega \\ 0 & x \in \mathcal{D}-\Omega\end{cases}
$$

where $\chi$ is the binary mask of the profile and $\boldsymbol{x}$ is the coordinate of a point on the Cartesian grid. The normal vector $\boldsymbol{n}$ is obtained via applying a convolution with a $3 \times 3$ Sobel operator kernel on $\chi$. Then, forces are calculated as

$$
\begin{gathered}
\boldsymbol{F}_{\text {pressure }}=\sum_{i \in \mathcal{D}-\otimes}(p \boldsymbol{n})_{i} \Delta l_{i} \\
\boldsymbol{F}_{\text {viscous }}=\sum_{i \in \mathcal{D}-\otimes}(\mu \boldsymbol{n} \times \boldsymbol{\omega})_{i} \Delta l_{i}
\end{gathered}
$$

where $i$ is the index of a point outside the profile and $\Delta l_{i}$ is the grid size at the point $i$. Thereby, $\operatorname{drag} \mathcal{L}$ is calculated using equation (4). As for the level set representation, the shape gradient $\frac{\partial \mathcal{L}}{\partial \mathbf{P}_{i}^{k}}$ is computed via automatic differentiation in order to drive the shape evolution to minimize $\mathcal{L}$.

\section{Neural network architecture and training pro- cedure}

\subsection{Architecture}

The neural network model is based on a U-Net architecture [51], a convolutional network originally used for the fast and precise segmentation of images. Following the methodology of previous work [18], we consider the inflow boundary conditions (i.e. $u_{\infty}, v_{\infty}$ ) and the shape of profiles (i.e. the binary mask) on the Cartesian grid $128 \times 128$ as three input channels. In the encoding part, 7 convolutional blocks are used to transform the input (i.e. $128^{2} \times 3$ ) into a single data point with 512 features. The decoder part of the network is designed symmetrically with another 7 layers in order to reconstruct the outputs with 


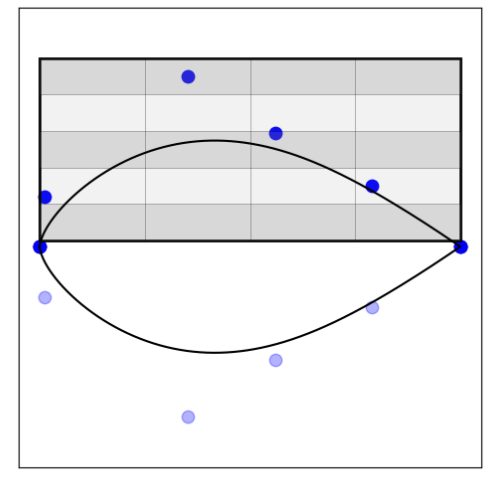

(a) Bézier control points

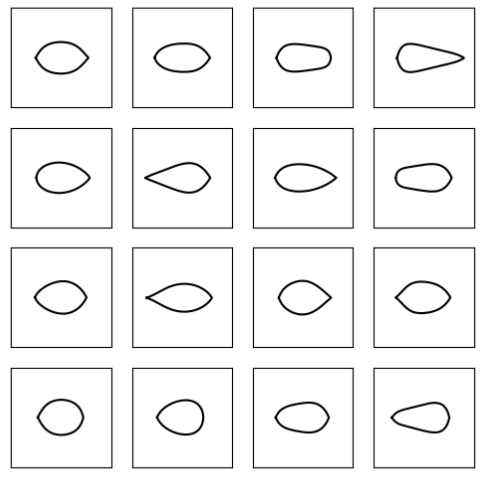

(b) Randomly generated shapes

Figure 3: Shape generation using two Bézier curves. The region of interest is divided into 4 columns, and each column-wise region is further split into 5 sub-regions.

the desired dimension, i.e. $128^{2} \times 3$, corresponding to the flowfield variables $[p, u, v]$ on the Cartesian grid $128 \times 128$. Leaky ReLU activation functions with a slope of 0.2 is used in the encoding layers, and regular ReLU activations in the decoding layers.

In order to assess the performance of the deep learning models, we have tested three different models with varying weight counts of $122 \mathrm{k}, 1.9 \mathrm{~m}$ and $30.9 \mathrm{~m}$, respectively, which are later referred to as small, medium and large-scale networks.

\subsection{Dataset generation}

For the training dataset, it is important to have a comprehensive coverage of the space of targeted solutions. In the present study, we utilize the parametric Bézier curve defined by equation (6) to generate randomized symmetric shape profiles subject to a fixed area constraint $S$.

To parameterise the upper surface of the profile, two points at the leading and trailing edges are fixed and 4 control points are positioned in different regions. As depicted in figure 3(a), the region of interest is divided into 4 columns separated by the border lines, and each control point of the upper Bézier curve is only allowed to be located within its corresponding column-wise region. Each column-wise region is further split into 5 sub-regions to produce diversified profiles. The subregions give $5^{4}=625$ possible permutations, with control points being placed randomly in each subregion. This procedure is repeated for 4 times, in total it produces $4 * 625=2500$ Bézier curves. Figure 


\begin{tabular}{lccc}
\hline Name & \# of flowfields & Re & NN models \\
\hline Dataset-1 & 2500 & 1 & small, medium \& large \\
Dataset-40 & 2500 & 40 & small, medium \& large \\
Dataset-Range & 8640 & $0.5-42.5$ & large \\
\hline
\end{tabular}

Table 1: Three datasets for training the neural network models.

3(b) shows some examples from this set.

Based on these 2500 geometries, we then generate three sets of training data, as summarised in table 1

(1) We run OpenFOAM with fixed $R e_{D}=1$ for all of the 2500 profiles to obtain 2500 flowfields, denoted as "Dataset-1".

(2) The second dataset is similar but all of the 2500 simulations are conducted at $R e_{D}=40$ ("Dataset-40").

(3) The third dataset is generated to cover a continuous range of Reynolds numbers, in order to capture a space of solutions that not only varies over the immersed shapes, but additionally captures a dimensions of varying flow physics with respect to a chosen Reynolds number. For this, we run a simulation by randomly choosing a profile $\Omega_{i}^{*}$ among 2500 geometries and a Reynolds number in the range of $R e_{D}^{*} \in[0.5,42.5]$. As we know that drag scales logarithmically w.r.t. Reynolds number, we similarly employ a logarithmic sampling for the Reynolds number dimension. We use a uniform distribution random variable $\kappa \in[\log 0.5, \log 42.5]$, leading to a $R e_{D}^{*}=10^{\kappa}$ uniformly distributed in $\log$ scale. In total we have obtained 8640 flowfields, which we refer to as "DatasetRange". With this size of the training dataset, the model performance converges to a stable prediction accuracy for training and validation losses, as shown in appendix A

As shown in figure 4 the distribution of all the flowfield samples from "Dateset-Range" on the $\Omega_{i}^{*}-R e_{D}^{*}$ map, with $R e_{D}^{*}$ in $\log$ scale. It worth noting that there are 2053 flowfield samples in the range of $R e_{D}^{*} \in[0.5,1.5]$ which are shown in red, 819 samples with $R e_{D}^{*} \in[8,12]$ colored in green and 195 samples with $R e_{D}^{*} \in[38,42]$ in blue.

\subsection{Pre-processing}

Proper pre-processing of the data is crucial for obtaining a high inference accuracy from the trained neural networks. Firstly, the nondimensional flowfield variables are calculated by

$$
\begin{array}{r}
\hat{p}_{i}=\left(p_{i}-p_{i, \text { mean }}\right) / U_{\infty, i}^{2}, \\
\hat{u}_{i}=u_{i} / U_{\infty, i}, \\
\hat{v}_{i}=v_{i} / U_{\infty, i} .
\end{array}
$$




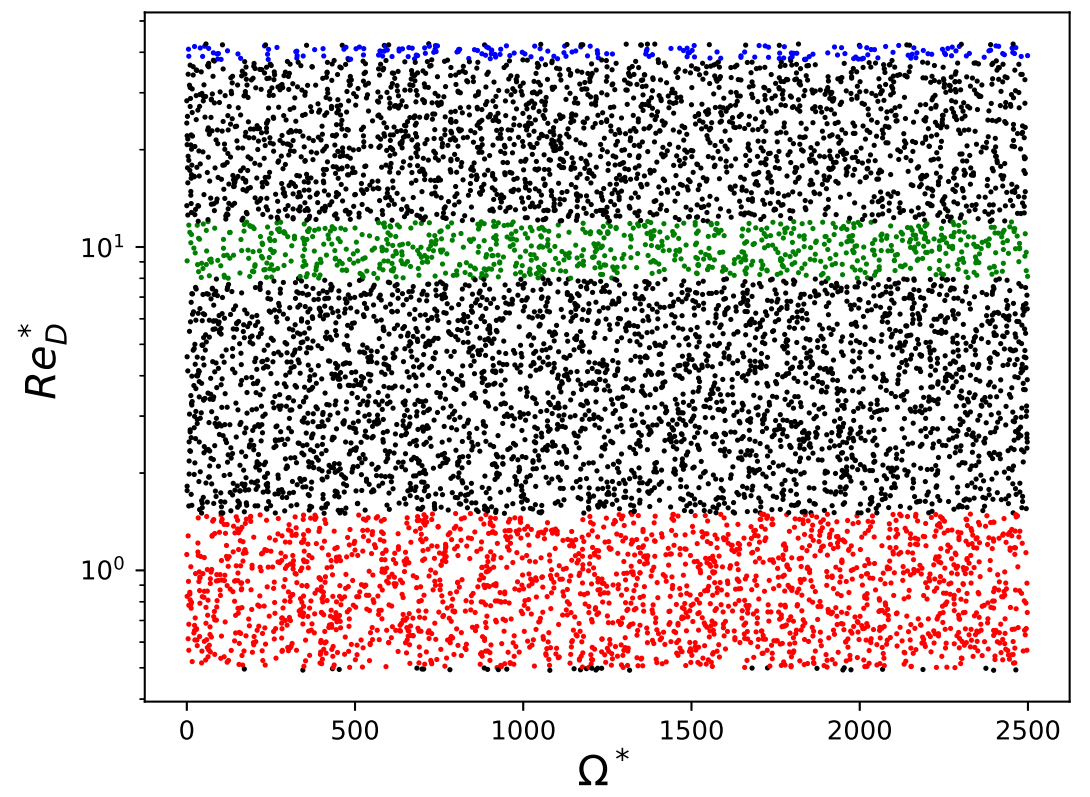

Figure 4: Distribution of flowfield samples from "Dataset-Range" on the $\Omega_{i}^{*}-$ $R e_{D}^{*}$ map. The indices of geometries $\Omega_{i}^{*}$ are from 0 to 2499 . The red symbols denote the flowfield samples with $R e_{D}^{*} \in[0.5,1.5]$, the green ones with $R e_{D}^{*} \in$ $[8,12]$ and the blue ones with $R e_{D}^{*} \in[38,42]$. 
Here, $i$ denotes the $i$-th flowfield sample in the dataset, $p_{\text {mean }}$ the simple arithmetic mean pressure, and $U_{\infty}=\sqrt{u_{\infty}^{2}+v_{\infty}^{2}}$ the magnitude of the freestream velocity.

As the second step, all input channels and target flowfield data in the training dataset are normalised to the range of $[-1,1]$ in order to minimise the errors from limited precision in the training phase. To do so, we need to find the maximum absolute values for each flow variable in the entire training dataset, i.e. $|\hat{p}|_{\max },|\hat{u}|_{\max }$ and $\left|\hat{v}_{\max }\right|$. Similarly, the maximum absolute values of the freestream velocity components are $\left|u_{\infty}\right|_{\max }$ and $\left|v_{\infty}\right|_{\max }$. Then we get the final normalised flowfield variables in the following form:

$$
\begin{aligned}
\tilde{p}_{i} & =\hat{p}_{i} /|\hat{p}|_{\text {max }} \\
\tilde{u}_{i} & =\hat{u}_{i} /|\hat{u}|_{\text {max }} \\
\tilde{v}_{i} & =\hat{v}_{i} /|\hat{v}|_{\max }
\end{aligned}
$$

and the normalised freestream velocities used for input channels are

$$
\begin{aligned}
\tilde{u}_{i} & =u_{i} / \max \left(\left|u_{\infty}\right|_{\max }, 1 \times 10^{-18}\right) \\
\tilde{v}_{i} & =v_{i} / \max \left(\left|v_{\infty}\right|_{\max }, 1 \times 10^{-18}\right)
\end{aligned}
$$

The freestream velocities appear in the boundary conditions, on which the solution globally depends, and should be readily available spatially and throughout the different layers. Thus, freestream conditions and the shape of the profile are encoded in a $128^{2} \times 3$ grid of values. The magnitude of the freestream velocity is chosen such that it leads to a desired Reynolds number.

\subsection{Training details}

The neural network is trained with the Adam optimiser in PyTorch [52]. A $L_{1}$ difference $L_{1}=\left|\mathbf{y}_{\text {truth }}-\mathbf{y}_{\text {prediction }}\right|$ is used for the loss calculation. For most of the cases, the training runs converge after 100k iterations with a learning rate $6 \times 10^{-4}$ and a batch size of 10 (unless otherwise mentioned). An $80 \%$ to $20 \%$ split is used for training and validation sets, respectively. The validation set allows for an unbiased evaluation of the quality of the trained model during training, for example, to detect overfitting. In addition, as learning rate decay is used, the variance of the learning iterations gradually decreases, which lets the training process fine-tune the final state of the model.

Figure 5 shows the training and validation losses for three models that are trained using "Dataset-1", i.e. small-scale, medium-scale and large-scale models, respectively. All the three models converge at stable levels of training and validation loss after 500 epochs. Looking at the training evolution for the smallscale model in figure 5(a), numerical oscillation can be seen in the early stage of the validation loss history, which is most likely caused by the smaller number of free parameters in the small-scale network. In contrast, the medium- and largescale models show a smoother loss evolution, and the gap between validation and training losses indicates a slight overfitting as shown in figures 5 (b) and 5 (c). 


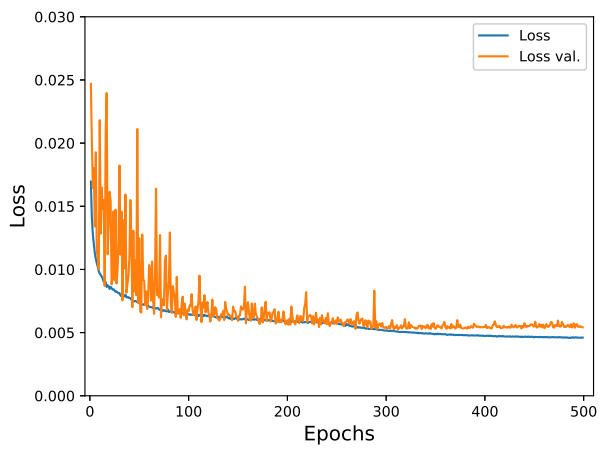

(a) Small-scale neural network

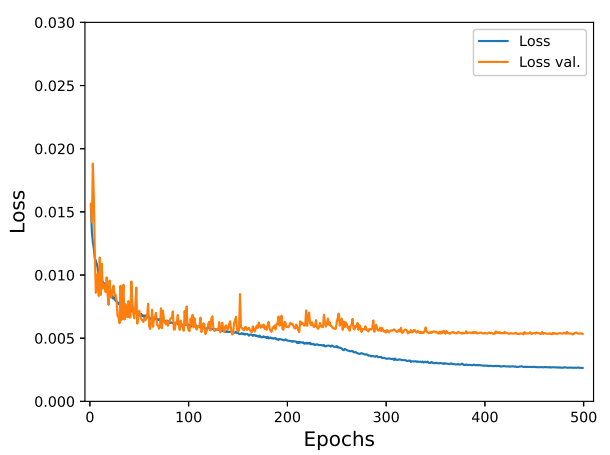

(b) Medium-scale neural network

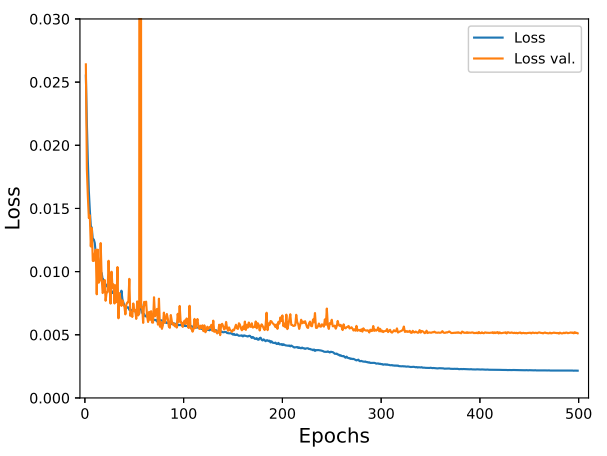

(c) Large-scale neural network

Figure 5: Training (in blue) and validation (in orange) losses of three different scales of models trained with "Dataset-1". 


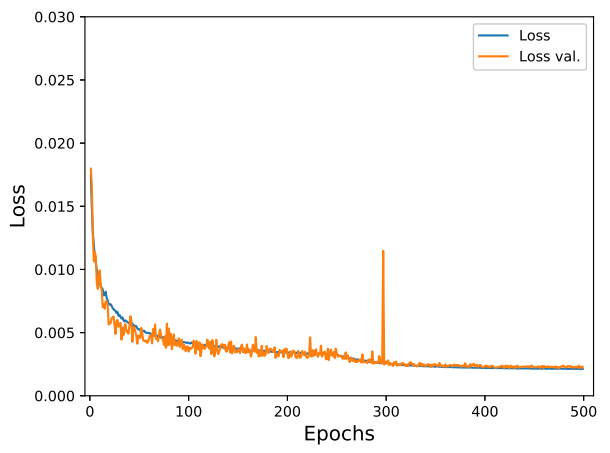

(a) Small-scale neural network

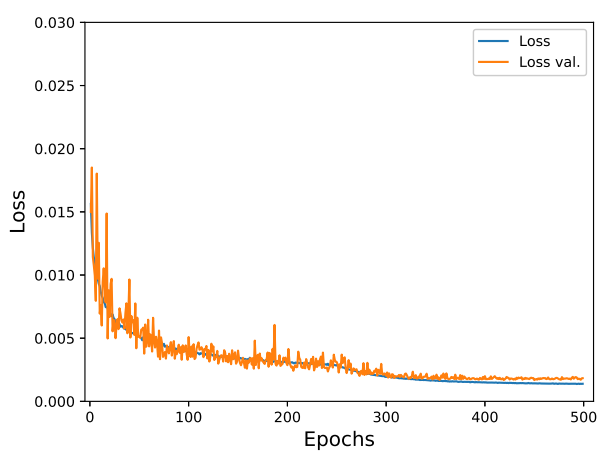

(b) Medium-scale neural network

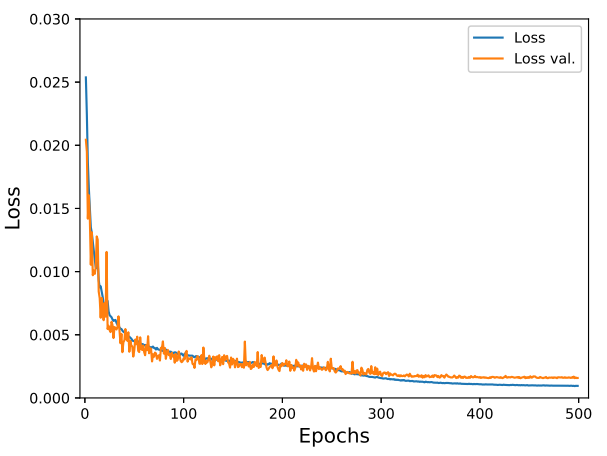

(c) Large-scale neural network

Figure 6: Training (in blue) and validation (in orange) losses of three different scales of models trained with "Dataset-40". 


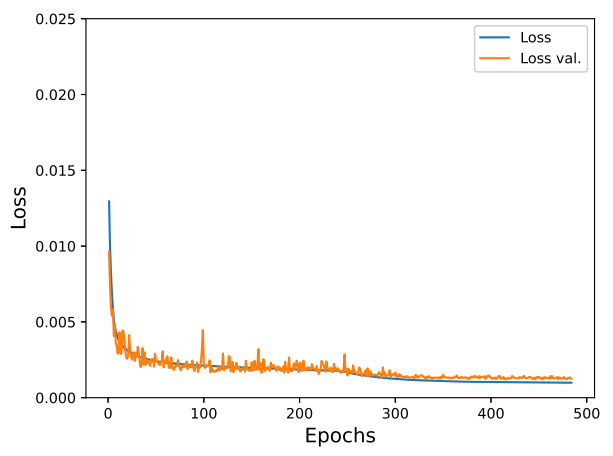

Figure 7: Training (in blue) and validation (in orange) losses of large-scale model trained with "Dataset-Range".

Although the training of the large-scale model exhibits a spike in the loss value at early stage due to an instantaneous pathological configuration of mini-batch data and learned state, the network recovers, and eventually the converges to lower loss values. Similar spikes can be seen in some of the other training runs, and could potentially be removed via gradient-clipping algorithms, which we, however, did not find necessary to achieve reliable convergence.

Figure 6 presents the training and validation losses for three models trained with "Dataset-40". Similarly, convergence can be achieved after 500 epochs. Compared to the training evolution at $R e_{D}=1$, the models $R e_{D}=40$ have smaller gaps between training and validation losses, indicating that the overfitting is less pronounced than for $R e_{D}=1$. We believe this is caused by the smoother and more diffusive flowfields at $R e_{D}=1$ (close to Stokes flow), in contrast to the additional complexity of the solutions at $R e_{D}=40$, which already exhibit separation bubbles.

We use "Dataset-Range" to train the model for a continuous range of Reynolds numbers. As this task is particularly challenging, we directly focus on the large scale network that has $30.9 \mathrm{~m}$ weights. To achieve better convergence for this case, we run $800 \mathrm{k}$ iterations with the batch size of 5 , which leads to more than 485 epochs. As shown in figure 7 training and validation losses converge to stable levels, and do not exhibit overfitting over the course of the training iterations. The final loss values are $1.01 \times 10^{-3}$ and $1.31 \times 10^{-3}$, respectively.

To summarise, having conducted the above-mentioned training, we obtain seven neural network models, i.e. models of three network sizes for "Dataset-1" and "Dataset-40" and a ranged model trained with "Dataset-Range" as list in table 1 These neural networks will be used as surrogate models in the optimisation in the next section. We will also compare the results from neural network models with corresponding optimisations conducted with the OpenFOAM solver, and evaluate the performance and accuracy of the optimisation runs. 


\section{Shape optimisation results}

The initial shape for the optimisation is a circular cylinder with a diameter $D \approx$ $0.39424[\mathrm{~m}]$. The integral value of the drag force using equation (4) is adopted as the objective function. The mathematical formula of the optimisation for the shape $\Omega$ bounded by curve $\Gamma$, the surface of the profile, is expressed as

$$
\begin{array}{r}
\min \operatorname{Drag}(\Omega) \\
\text { subject to Area } S(\Omega)=S_{0} \\
\text { Barycenter } \mathbf{b}(\Omega)=\frac{1}{S(\Omega)} \int_{\Omega} \boldsymbol{x} d s=(0,0)
\end{array}
$$

For the level-set representation, the profile $\Omega$ is the region where $\phi \leq 0$ and the constrained optimisation problem is solved as follows:

(1) Initialise level set function $\phi$ such that the initial shape (i.e. a circular cylinder) is corresponding to $\phi=0$.

(2) For a given $\phi$, calculate drag (i.e. loss $\mathcal{L}$ ) using Equations (24). Terminate if the optimisation converges, e.g. drag history reaches a statistically steady state.

(3) Calculate the gradient $\frac{\partial \mathcal{L}}{\partial \phi}$. Consider an unconstrained minimisation problem and solve equation (5) as follows:

$$
\phi^{n+1} \Longleftarrow \phi^{n}-\Delta \tau \frac{\partial \mathcal{L}}{\partial \phi}|\nabla \phi|
$$

In practice, we update $\phi$ using the second-order Runge-Kutta method, and discretise the convection term with a first-order upwind scheme [43]. We assume derivatives of the flowfield variables (i.e. pressure and velocity) are significantly smaller than those w.r.t. to the shape. Hence, we treat both fields as constants for each step of the shape evolution. To ensure the correct search direction for optimisation, we use a relatively small pseudo time step $\Delta \tau$, which is calculated with a CFL number of 0.8 .

(4) To ensure $|\nabla \phi| \approx 1$, a fast marching method is used to solve the Eikonal equation [46].

(5) The area of the shape $\Omega$ is obtained by $S=\int_{\mathcal{D}} H_{\epsilon}(-(\phi+\eta)) d s$, where $\eta$ is an adjustable constant. We optimise $\eta$ such that $\left|S-S_{0}\right|<\epsilon$. Then, we update $\phi^{n+1} \Longleftarrow \phi^{n+1}+\eta$.

(6) Check if the barycenter is at the origin: $|\mathbf{b}-\mathbf{o}|<\epsilon$. If not, solve equation (5) to update $\phi^{n+1}$ by replacing $V_{n}$ with a translating constant velocity so that the barycenter of the shape $\Omega$ moves towards the origin. Continue with (2).

While our main focus lies on level-set representations, the Bézier curve parameterisation with reduced degrees of freedom are used for comparison purposes. They highlight how differences in the shape parameterisation can influence the optimisation results. Thus, we include the Bezier parameterisation with very few control points and the level-set representation with a dense grid sampling as two extremes of the spectrum of shape representations. When Bézier curves are used, the constrained optimisation defers from the above-mentioned 


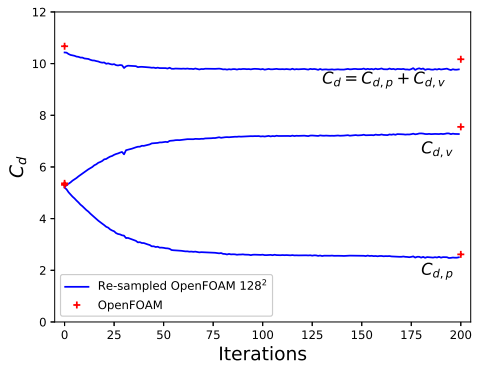

(a) OpenFOAM

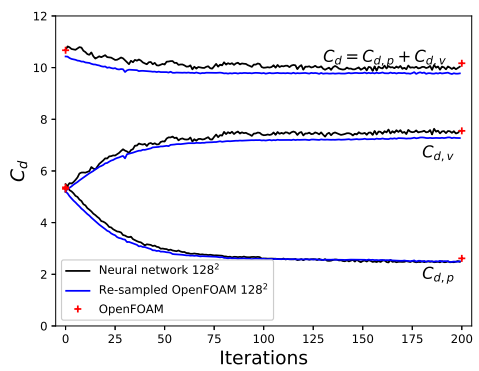

(c) Medium-scale neural network

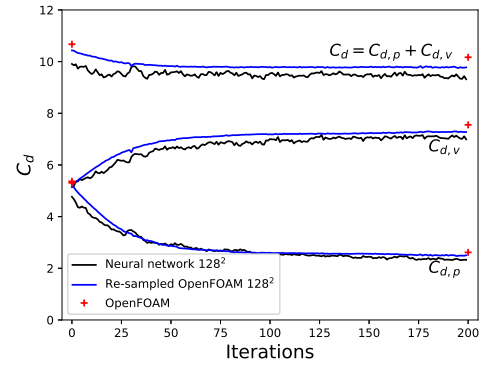

(b) Small-scale neural network

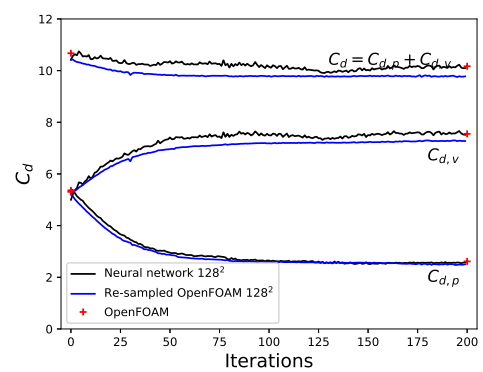

(d) Large-scale neural network

Figure 8: Optimisation histories at $R e_{D}=1$. The black solid lines denote the results using neural network models trained with "Dataset-1" and the blue solid lines denote the results from OpenFOAM. Results calculated with the resampled flowfields on the $128 \times 128$ Cartesian grid are denoted by $128^{2}$. The red cross symbols represent the OpenFOAM's results obtained with its native postprocessing tool.

loop in the following: In (1-3), the coordinates of Bézier curve control points are used as the design variables to be initialised and updated. In (5) and (6), the area of $\Omega$ and barycenter are calculated based on the region enclosed by the Bézier curve, where the binary mask of inner region is 1 and outer region is 0 .

In the optimisation experiments the flowfield solvers used are OpenFOAM (as baseline), small, medium and large-scale neural network models, respectively. As additional validation for the optimisation procedure, we also compare to additional runs based on the Bézier curve parameterisation with a large-scale neural network model. If the flow solver is OpenFOAM, Gmsh is automatically called to generate an unstructured mesh based on the curve $\phi=0$ at every iteration. To update $\phi$ and calculate drag in Step (2), as aforementioned in \$2.1. the flowfield variables are re-sampled on the $128 \times 128$ Cartesian grid. 


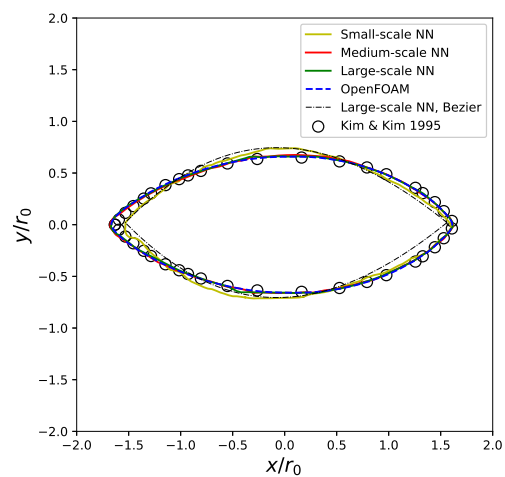

(a)

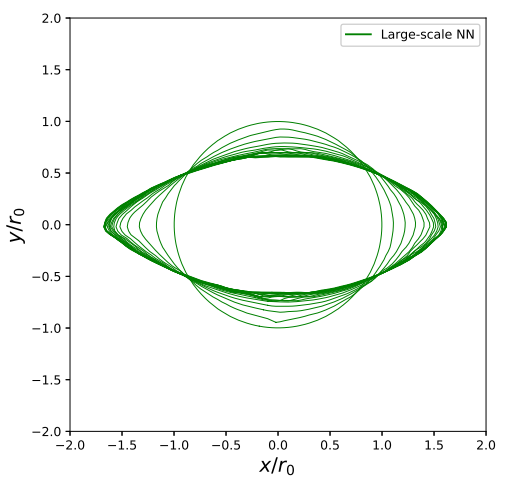

(b)

Figure 9: The converged shapes at $R e_{D}=1$ (a) and the intermediate states at every 10th iteration by large-scale NN model (b).

\subsection{Optimisation experiment at $R e_{D}=1$}

Figures 8 (a-d) present the drag coefficients over 200 optimisation iterations using OpenFOAM solver and three neural network models. Here, the drag coefficient $C_{d}$ is defined as drag divided by the projected length of the initial cylinder and dynamic head. The same definition is used for all of other experiments in the present paper. As the ground truth, figure 8(a) shows the case which uses the OpenFOAM solver in the optimisation. The history of drag values, shown in blue, is calculated based on the re-sampled data on the Cartesian grid (i.e. $128^{2}$ ). For comparison, the drag values obtained from the surface integral in the OpenFOAM's native post-processing code are shown with red markers. As can be seen in figure 8(a), after convergence of the optimisation the total drag drops $6.3 \%$ from 10.43 to 9.78 . To further break it down, the inviscid part decreases significantly from 5.20 to $2.50(\sim 51.8 \%)$ while the viscous part gradually increases from 5.23 to $7.27(\sim 31.0 \%)$. This is associated with the elongation of the shape from a circular cylinder to an "oval", eventually becoming a rugby shape as shown in figure 9(b).

From figures $8(b-c)$, one can observe the histories of the drag values are reasonably well predicted by neural network models and agree with the OpenFOAM solution in figure 8(a). Despite the small scale model exhibiting noticeable oscillations in the optimisation procedure, the medium and large-scale neural network models provide smoother predictions, and the drag of both initial and final shapes agrees well with that from re-sampled data (blue lines) and the one from OpenFOAM's native post-processing code (red symbols).

Figure 9(a) depicts the converged shapes of all four solvers. The ground truth result using OpenFOAM ends up with a rugby shape which achieves a good agreement with the data by [30]. The medium and large-scale neural 


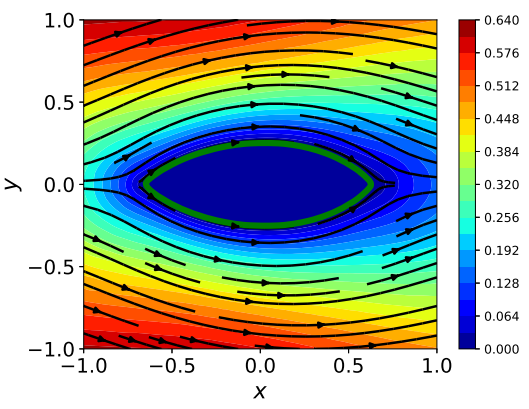

(a) OpenFOAM

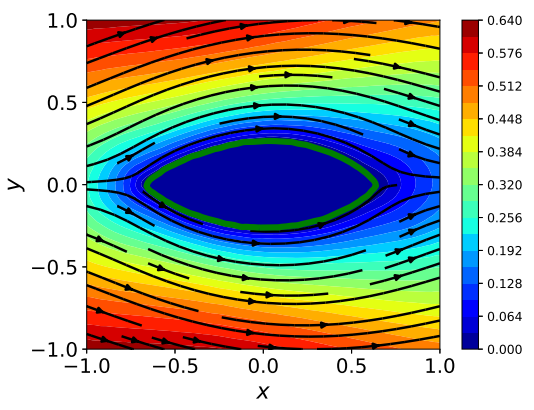

(c) Medium-scale neural network

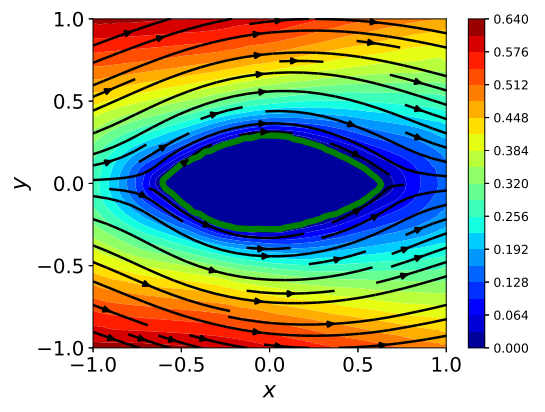

(b) Small-scale neural network

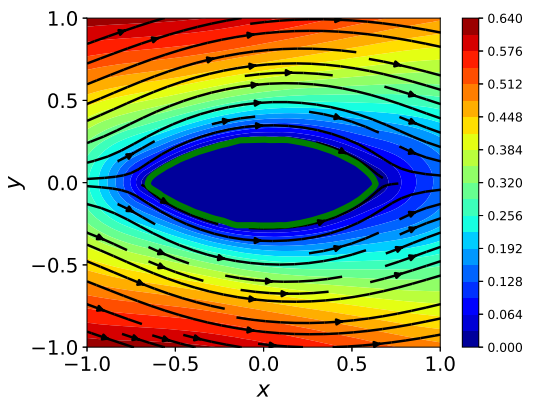

(d) Large-scale neural network

Figure 10: Streamlines and the x-component velocity fields $u / U_{\infty}$ at $\operatorname{Re}_{D}=1$.

network models collapse and compare favourably with the ground truth result. In contrast, the small-scale neural network model's prediction is slightly off which is not surprising as one can observe oscillation and offset of the drag history in figure 8(b) as discussed before. A possible reason is that the small scale model has less weights so that the complexity of the flow evolution cannot be fully captured. It is worth noting that the reduced performance of the Bézier representation in the present work is partly due to the discretization errors when calculating the normal vectors in combination with a reduced number of degrees of freedom.

The x-component velocity fields with streamlines for the optimised shapes are shown in figure 10. The flowfields and the patterns of streamlines in all the three cases with neural networks show no separation, which is consistent with the ground truth result in figure 10(a). Considering the final shape obtained using the three neural network surrogates, the medium- and large-scale models give satisfactory results that are close to the OpenFOAM result.

\subsection{Optimisation experiment at $R e_{D}=40$}

As the Reynolds number increases past the critical Reynolds number $\operatorname{Re}_{D} \approx 47$, the circular cylinder flow configuration loses its symmetry and becomes unstable, 


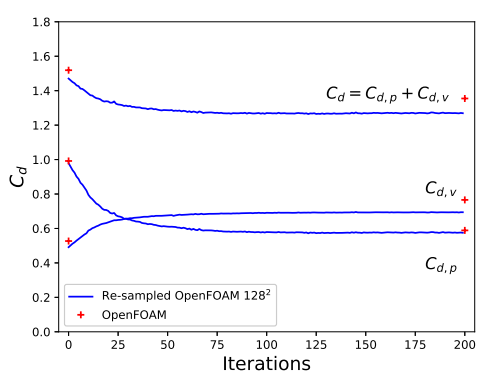

(a) OpenFOAM

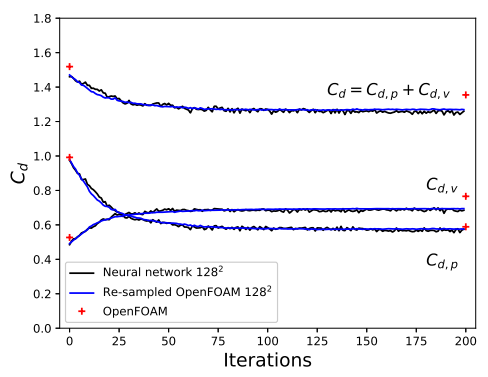

(c) Medium-scale neural network

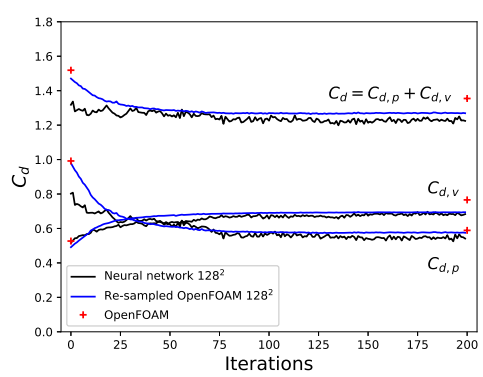

(b) Small-scale neural network

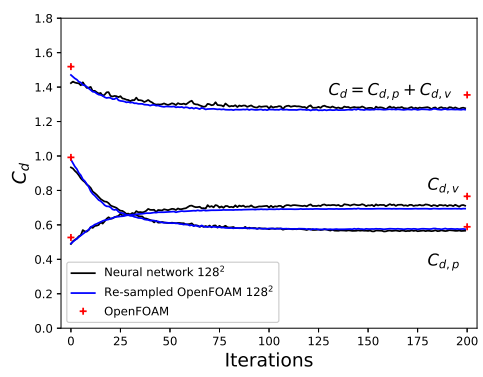

(d) Large-scale neural network

Figure 11: Optimisation histories at $R e_{D}=40$. The black solid lines denote the results using neural network models trained with "Dataset-40" and the blue solid lines denote the results from OpenFOAM. Results calculated with the resampled flowfields on the $128 \times 128$ Cartesian grid are denoted by $128^{2}$. The red cross symbols represent the OpenFOAM's results obtained with its native postprocessing tool. 


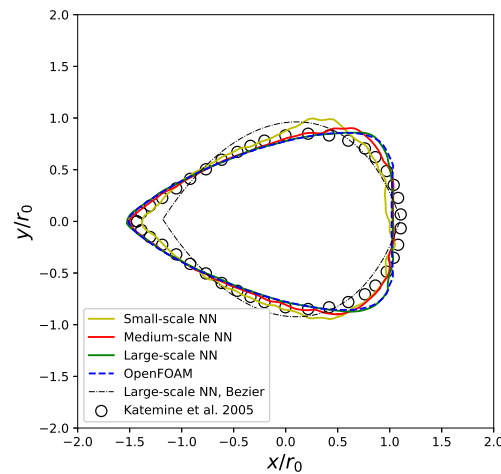

(a)

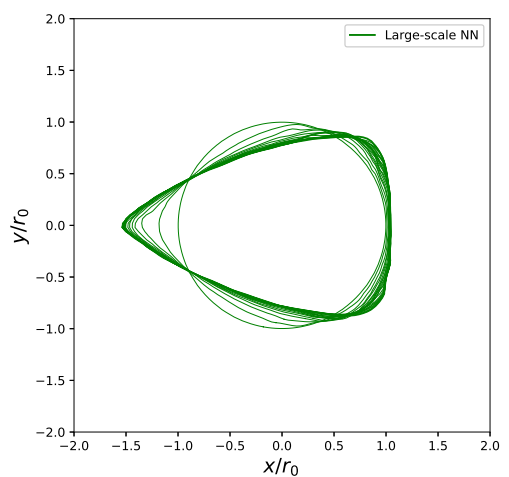

(b)

Figure 12: The converged shapes at $\operatorname{Re}_{D}=40$ (a) and the intermediate states at every 10th iteration by large-scale NN model (b).

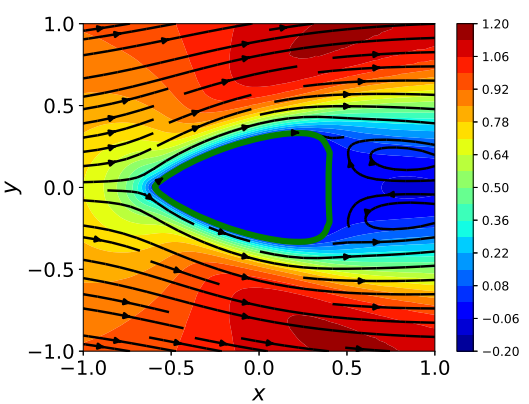

(a) OpenFOAM

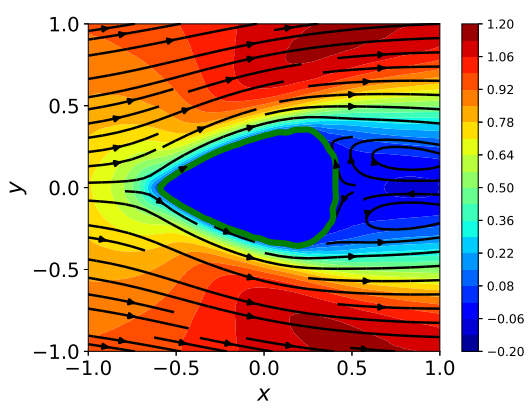

(c) Medium-scale neural network

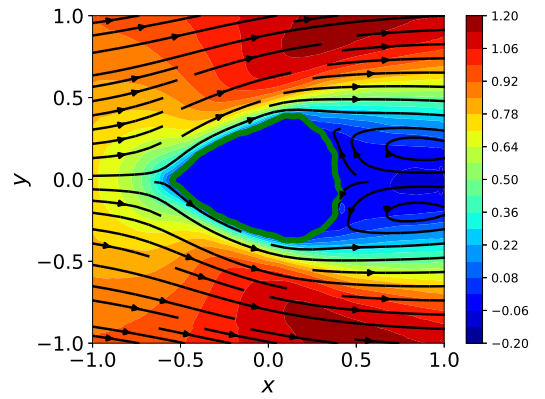

(b) Small-scale neural network

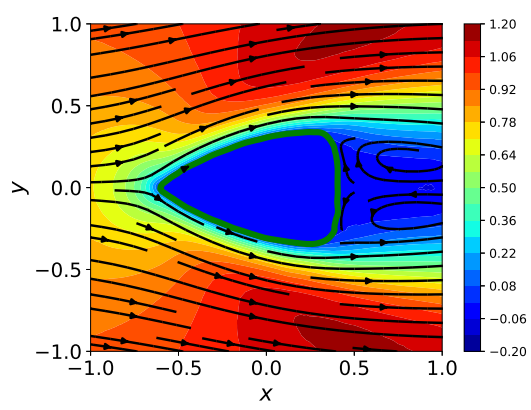

(d) Large-scale neural network

Figure 13: Streamlines and the x-component velocity fields $u / U_{\infty}$ at $R e_{D}=$ 40 obtained with different solvers, i.e. OpenFOAM, and three neural network models trained with "Dataset-40". 
which is known as the Karman vortex street. We consider optimisations for the flow regime at $R e_{D}=40$ which is of particular interest because it exhibits a steady-state solution, yet is close to the critical Reynolds number. The steady separation bubbles behind the profile further compound the learning task and the optimisation, making it a good test case for the proposed method.

The ground truth optimisation result using OpenFOAM is shown in figure 11(a). The shape is initialised with a circular cylinder and is optimised to minimise drag over 200 iterations. As a result, the total drag, processed on the Cartesian grid, drops from 1.470 to 1.269 ( 13.7\% reduction). Associated with the elongation of the shape, the inviscid drag decreases $41.3 \%$ while the viscous drag increases $41.3 \%$. The initial and the final results of the OpenFOAM's native post-processing are shown in red, indicating good agreement. Figures 11(b-d) present the drag histories over 200 optimisation iterations with three neural network models that are trained with "Dataset-40". Although larger oscillations are found in the drag history of the small scale model, the medium and large scale models predict smoother drag history and compare well with the ground truth data using OpenFOAM.

The final converged shapes are compared to a reference result [31] in figure 12(a). The evolution of intermediate shapes from the initial circular cylinder towards the final shape is shown in figure 12(b). The upwind side forms a sharp leading edge while the downwind side of the profile develops into a blunt trailing edge. Compared to the reference data [31] and the result using Bézier-curve based method, the use of level-set based method leads to a slightly flatter trailing edge, probably because more degrees of freedom for the shape representation are considered in level-set based method.

Further looking at the details of shapes in figure 13, it can be seen that the more weights the neural network model contains, the closer it compares to the ground truth result using OpenFOAM. The large scale model which has the largest weight count is able to resolve the fine feature of the flat trailing edge as shown in figure 13(d). In contrast, in figure 13(b), the small scale model does not capture that and even the the surface of the profile exhibits pronounced roughness. Nonetheless, all the three DNN models predict similar flow patterns compared to the ground truth result depicted with streamlines, which are characterised with re-circulation regions downstream of the profiles.

It should be mentioned that the optimised shape at $R e_{D}=40$ by Kim and Kim [30] differs from the one in the present study and the one by Katamine et al. [31]. In the former [30], the optimised profile converges at an elongated slender shape with an even smaller drag force. Most likely, this is caused by that an additional wedge angle constraint is imposed at both leading and trailing edge, which is not adopted in our work and Katamine et al. [31]. As we focus on deep learning surrogates in the present study, we believe the topic of including additional constraints will be an interesting avenue for future work. In the comparison to the ground truth from OpenFOAM, the current results are deemed to be in very good agreement. 


\subsection{Shape optimisations for an enlarged solution space}

The generalising capabilities of neural networks are a challenging topic [53]. To evaluate their flexibility in our context, we target shape optimisations in the continuous range of Reynolds numbers from $R e_{D}=1$ to 40 , over the course of which the flow patterns change significantly [40,54]. Hence, in order to succeed, a neural network not only has to encode change of the solutions w.r.t. immersed shape but also the changing physics of the different Reynolds numbers. In this section, we conduct four tests at $R e_{D}=1,5,10$, and 40 with the ranged model in order to quantitatively assess the its ability to make accurate flowfield predictions over the chosen change of Reynolds numbers. The corresponding OpenFOAM runs are used as ground truth for comparisons.

The optimisation histories for the four cases are plotted in figures 14(a-d). Despite some oscillations, the predicted drag values as well as the inviscid and viscous parts agree well with the ground truth values from OpenFOAM. The total drag force as objective function has been reduced and reaches a stable state in each case. The performance of the ranged model at $R e_{D}=40$ is reasonably good, although it is slightly outperformed by the specialized NN model trained with "Dataset-40".

In line with the previous runs, the overall trend of optimisation for the four cases shows that the viscous drag increases while the inviscid part decreases as shown in figures 14(a-d), which is associated with an elongation of the profile and the formation of the sharp leading edge. The final shapes after optimisation for four Reynolds numbers are summarised in figure 15. For the four cases, the leading eventually develops a sharp leading edge, while the trailing edge shows difference. At $R e_{D}=1$ and 5 , the profiles converge with sharp trailing edges as depicted in figures 15(a) and 15(b). The corresponding flowfields also show no separations in figures 16(a) and 16(b).

As shown in figure 15(c) at $R e_{D}=10$ and figure 15(d) at $R e_{D}=40$, blunt trailing edges become the final shapes and the profile at $R e_{D}=10$ is more slender than that for $R e_{D}=40$. The higher Reynolds number leads to a flattened trailing edge, associated with the occurrence of the recirculation region shown in figures 16(c) and 16(d), and the gradient of the objective function becoming relatively week in these regions. In terms of accuracy, the converged shapes at $R e_{D}=1,5$, and 10 compare favourably with the results with OpenFOAM. Compared to ground truth shapes, only the final profile at $R e_{D}=40$ predicted by the ranged model shows slight deviations near trailing edge. Thus, given the non-trivial changes of flow behavior across the targeted range or Reynolds numbers, the neural network yields a robust and consistent performance.

\subsection{Performance}

The performance of trained deep neural network models is one of the central factors motivating their use. We evaluate our models in a standard workstation with 12 cores, i.e. Intel(R) Xeon(R) W-2133 CPU @ 3.60GHz, with an NVidia GeForce RTX 2060 GPU. The optimisation run at $R e_{D}=1$ which consists of 


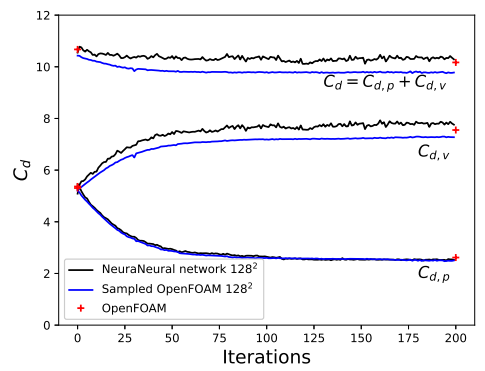

(a) $R e_{D}=1$

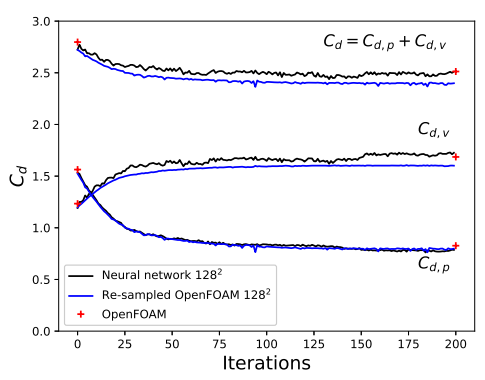

(c) $\operatorname{Re}_{D}=10$

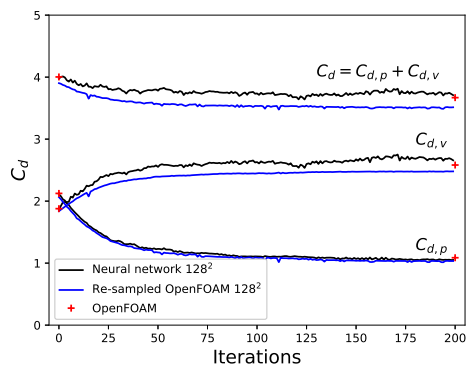

(b) $R e_{D}=5$

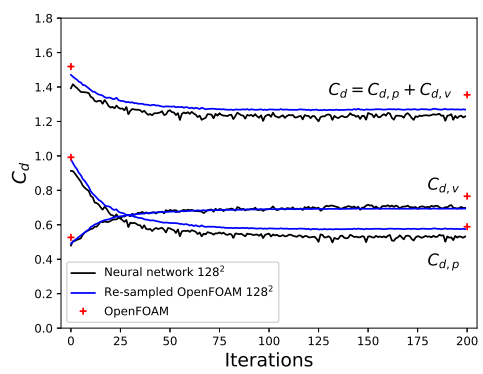

(d) $R e_{D}=40$

Figure 14: Optimisation history for the four cases at $R e_{D}=1,5,10$, and 40 . The black solid lines denote the results using neural network models (i.e. the ranged model) and the blue solid lines denote the results from OpenFOAM. Results calculated with the re-sampled flowfields on the $128 \times 128$ Cartesian grid are denoted by $128^{2}$. The red cross symbols represent the OpenFOAM results obtained with its native postprocessing tool.

\begin{tabular}{lll}
\hline Solver & Wall time & Platform \\
\hline OpenFOAM & $16.3 \mathrm{hr}$ & CPU only, 9 cores \\
Small-scale DNN & $97 \mathrm{sec}$ & CPU, 1 core \& GPU \\
Medium-scale DNN & $106 \mathrm{sec}$ & CPU, 1 core \& GPU \\
Large-scale DNN & $196 \mathrm{sec}$ & CPU, 1 core \& GPU \\
\hline
\end{tabular}

Table 2: Run times for 200 optimisation iterations at $R e_{D}=1$. 


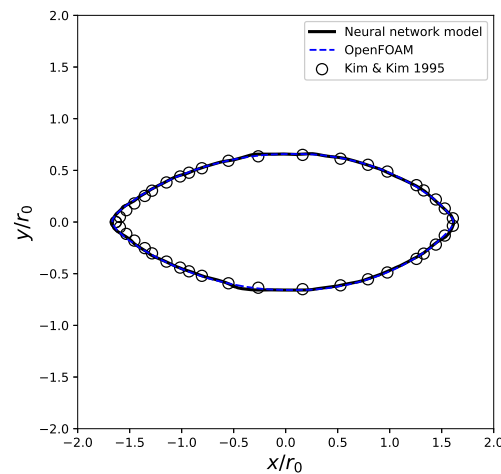

(a) $R e_{D}=1$

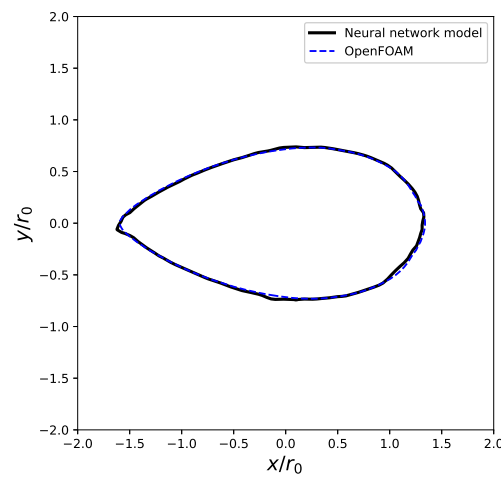

(c) $R e_{D}=10$

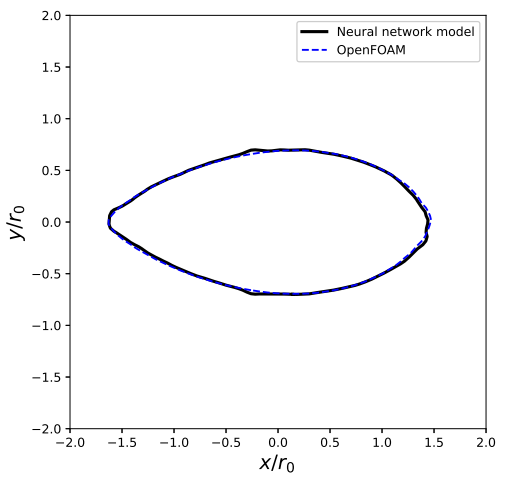

(b) $R e_{D}=5$

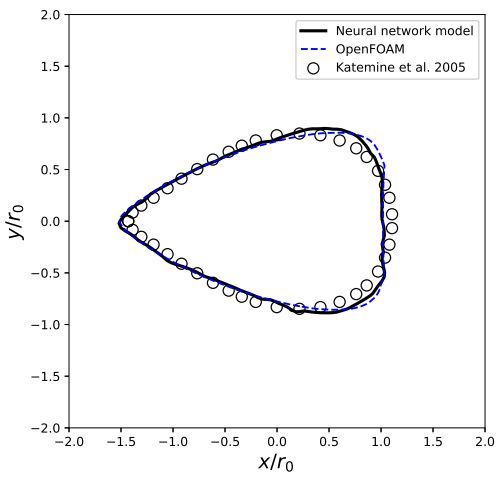

(d) $R e_{D}=40$

Figure 15: Shapes after optimisation at $R e_{D}=1,5,10$, and 40. The black solid lines denote the results using neural network models (i.e. the ranged model), the blue dashed lines denote the results from OpenFOAM and the symbols denote the corresponding reference data. 


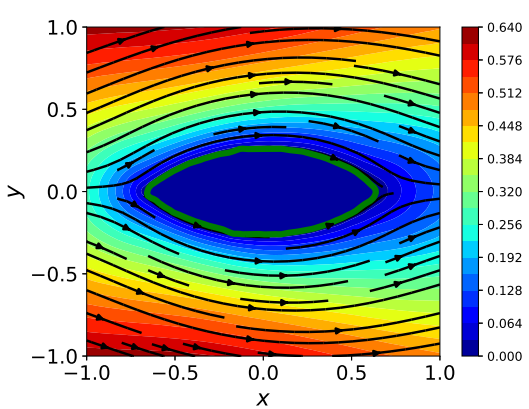

(a) $R e_{D}=1$

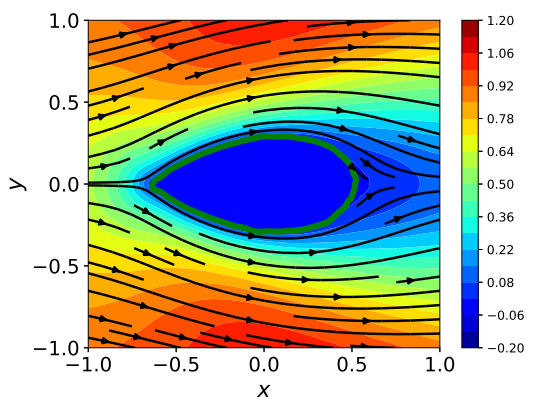

(c) $R e_{D}=10$

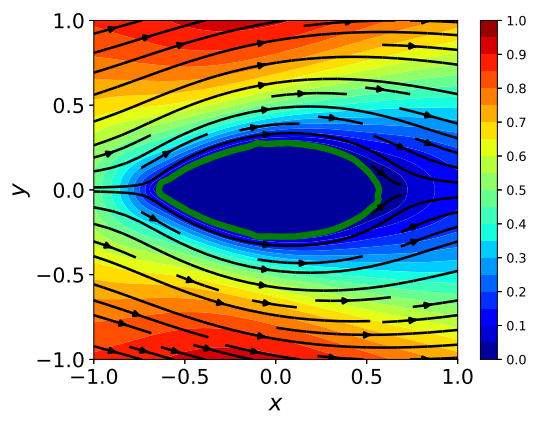

(b) $R e_{D}=5$

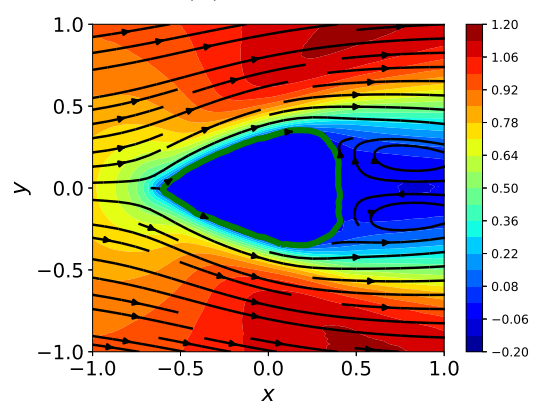

(d) $R e_{D}=40$

Figure 16: Streamlines and the x-component velocity fields $u / U_{\infty}$ at $R e_{D}=1$, 5,10 , and 40 using the ranged model. 
200 iterations is chosen for evaluating the run times using different solvers, i.e. OpenFOAM, DNN models of three sizes trained with "Dataset-1". Due to the strongly differing implementations, we compare the different solvers in terms of elapsed wall clock time. As listed in table 2 it takes 16.3 hours using 9 cores (or 147 core-hours) for OpenFOAM to complete such a case. Compared to OpenFOAM, the DNN model using the GPU reduces the computational cost significantly. The small-scale model requires 97 seconds and even the large-scale model only takes less than 200 seconds to accomplish the task. Therefore, relative to OpenFOAM, the speed-up factor is between $600 \mathrm{X}$ and $300 \mathrm{X}$. Even when considering a factor of ca. 10 in terms of GPU advantage due to an improved on-chip memory bandwidth, these measurements indicate the significant reductions in terms of runtime that can potentially be achieved by employing trained neural networks.

The time to train the DNN models varies with neural network size and the amount of training data. Taking "Dataset-1" as an example, the time of training start with 23 minutes for the small-scale model, up to 124 minutes for the largescale model. When using "Dataset-Range" (8640 samples), it takes 4 hours and 12 minutes to train a large-scale ranged model.

Note that we aim for providing a possible choice rather than down-playing the alternatives, e.g. optimisation using discrete adjoints [9]. Given the potentially large one-time cost for training a model, learned approaches bear particular promise in settings where similar optimisation problems need to be solved repeatedly. Considering the cost of data generation and training, we also believe that hybrid methods that combine deep-learning and traditional numerical methods pose very promising avenues for future algorithms. E.g., to employ learned models as fast initialisers for non-linear optimisations [25].

\section{Concluding remarks}

In this paper, deep neural networks are trained to infer flowfields, and used as surrogate models to carry out shape optimisation for drag minimisation of the flow past a profile with a given area subjected to the two-dimensional incompressible fluid at low Reynolds numbers. Both level-set and Bézier curve representations are adopted to parameterise the shape, the integral values on the re-sampled Cartesian mesh are used as the optimisation objective. The gradient flow that drives the evolution of shape profile is calculated by automatic differentiation in a deep learning framework, which seamlessly integrates with trained neural network models.

Through optimisation, the drag values predicted by neural network models agree well with the OpenFOAM results showing consistent trends. Although the total drag decreases, it is observed that the inviscid drag decreases while the contribution of viscous part increases, which is associated with the elongation of the shape. It is demonstrated that the present DNN model is able to predict satisfactory drag forces and the proposed optimisation framework is promising to be used for general aerodynamic design. Moreover, the DNN model stands 
out with respect to its flexibility, as it predicts a full flowfield in a region of interest and, once trained, can potentially be used in other optimization tasks with multiple objectives. In conjunction with the low run-time of the trained deep neural network, we believe the proposed method showcases the possibilities of using deep neural networks as surrogates for optimisation problems in physical sciences.

\section{Acknowledgements}

The authors are grateful to Oguzhan Karakaya and Hao Ma for the valuable discussions. This work was supported by European Research Council (ERC) grants 637014 (realFlow), and 838342 (dataFlow).

\section{Declaration of interests}

The authors report no conflict of interest.

\section{A Appendix}

To assess the effects of the number of samples in the datasets on the training and validation losses, six training runs are conducted with various amounts of data, which are listed in table A1. The method to generate those datasets has been discussed in $\$ 3.2$. Note that in table A1 any smaller dataset is a subset of a larger dataset and all follow the same probability distribution (see figure 4).

We found validation sets of several hundred samples to yield stable estimates, and hence use an upper limit of 400 as the maximal size of the validation dataset.

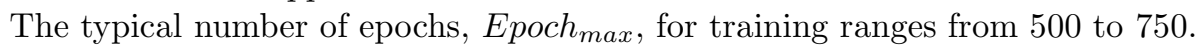

In figures A1(a) and A1(b), all models converge to stable levels of training and validation losses, and do not exhibit overfitting despite the reduced amount of data for some of the runs. For all graphs, the onset of learning rate decay can be seen in the middle of the plot. It can be seen that with an increased number of samples, the training and validation losses follow an overall declining trend, and the variance of training losses noticeably decreases. Additionally, looking at the models trained with "Dataset-Range-5815" and "Dataset-Range-8640", the two curves are very close in terms of the loss values, which implies that further increasing the amount of data does not yield significant improvements in terms of inference accuracy.

Figure A2 shows the values of training and validation losses (averaged in the last 100 epochs). It can be observed that the models with small amounts of data exhibit larger losses. Both training and validation loss curves show drastic drops over the course of the first data points with small amounts of data. This indicates that adding samples leads to remarkable improvements when the data amount is smaller than 3000. The behavior stabilizes with larger amounts of 


\begin{tabular}{lccc}
\hline Name & \# of flowfields & training & validation \\
\hline Dataset-Range-400 & 400 & 320 & 80 \\
Dataset-Range-800 & 800 & 640 & 160 \\
Dataset-Range-1660 & 1660 & 1330 & 330 \\
Dataset-Range-3315 & 3315 & 2915 & 400 \\
Dataset-Range-5815 & 5815 & 5415 & 400 \\
Dataset-Range-8640 & 8640 & 8240 & 400 \\
\hline
\end{tabular}

Table A1: Different dataset sizes used for training runs with corresponding splits into training and validation sets.

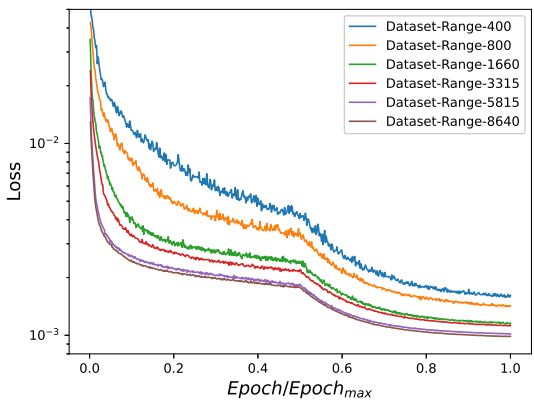

(a) Training loss

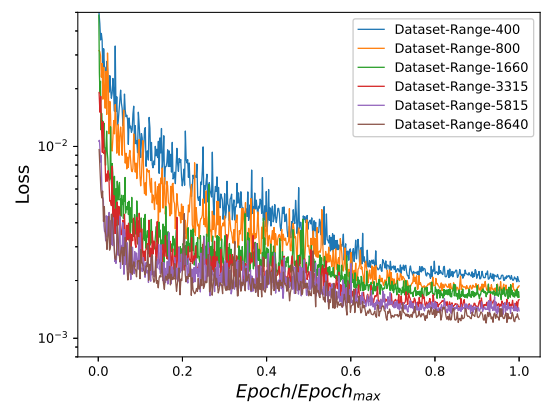

(b) Validation loss

Figure A1: Comparison of training histories for different training data amounts.

data being available for training, especially when the number of samples is greater than 5000 .

Based on the above test results, "Dataset-Range-8640" is chosen for the optimisation study at the Reynolds number range $R e \in[0,40]$ above. For the sake of brevity, "Data-Range" is used to denote this dataset in the main text.

\section{References}

[1] D. M. Bushnell and K. J. Moore. Drag reduction in nature. Annu. Rev. Fluid Mech., 23(1):65-79, 1991. doi: 10.1146/annurev.fl.23.010191.000433. URL https://doi.org/10.1146/annurev.fl.23.010191.000433.

[2] D. M. Bushnell. Aircraft drag reduction - a review. J. Aerospace Eng., 217 (1):1-18, 2003. doi: 10.1243/095441003763031789.

[3] D. Thévenin and G. Janiga, editors. Optimization and Computational Fluid Dynamics, chapter 16, 17. Springer-Verlag Berlin Heidelberg, Berlin, Germany, 2008. 


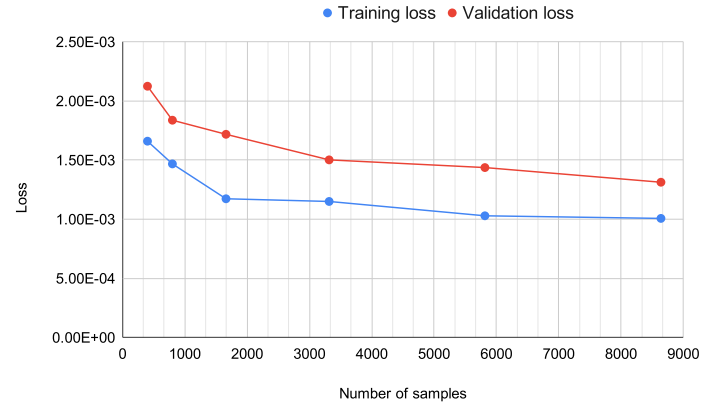

Figure A2: Training and validation losses (L1) for different training data amounts.

[4] S. N. Skinner and H. Zare-Behtash. State-of-the-art in aerodynamic shape optimisation methods. Appl. Soft Comput., 62:933-962, 2018. ISSN 1568-4946. doi: https://doi.org/10.1016/j.asoc.2017.09.030. URL http://www.sciencedirect.com/science/article/pii/S1568494617305690

[5] A. Jameson. Aerodynamic design via control theory. Journal of Scientific Computing, 3:233-260, 1988. doi: https://doi.org/10.1007/BF01061285.

[6] M. B. Giles and N. A. Pierce. An introduction to the adjoint approach to design. Flow, Turbul. Combust., 65:393-415, 2000. doi: https://doi.org/ 10.1023/A:1011430410075.

[7] T. D. Economon, F. Palacios, and J. J. Alonso. A Viscous Continuous Adjoint Approach for the Design of Rotating Engineering Applications. In 21st AIAA Computational Fluid Dynamics Conference. American Institute of Aeronautics and Astronautics, 2013. doi: 10.2514/6.2013-2580. URL https://doi.org/10.2514/6.2013-2580.

[8] H. L. Kline, T. D. Economon, and J. J. Alonso. Multi-Objective Optimization of a Hypersonic Inlet Using Generalized Outflow Boundary Conditions in the Continuous Adjoint Method. In 54th AIAA Aerospace Sciences Meeting, 2016. doi: doi:10.2514/6.2016-0912.

[9] B. Y. Zhou, T. Albring, N. R. Gauger, C. R. Ilario da Silva, T. D. Economon, and J. J. Alonso. An Efficient Unsteady Aerodynamic and Aeroacoustic Design Framework Using Discrete Adjoint. AIAA Paper 2016-3369, July 2016. URL https://arc.aiaa.org/doi/10.2514/6.2016-3369

[10] L. Mueller and T. Verstraete. Adjoint-based multi-point and multiobjective optimization of a turbocharger radial turbine. Int. J. Turbomach. Propuls. Power, 2:14-30, 2019. doi: 10.3390/ijtpp4020010. 
[11] D. W. Zingg, M. Nemec, and T. H. Pulliam. A comparative evaluation of genetic and gradient-based algorithms applied to aerodynamic optimization. Eur. J. Comput. Mech., 17(1-2):103-126, 2008. doi: 10.3166/remn. 17.103-126. URL https://doi.org/10.3166/remn.17.103-126.

[12] N. V. Queipo, R. T. Haftka, W. Shyy, T. Goel, R. Vaidyanathan, and P. Kevin Tucker. Surrogate-based analysis and optimization. Prog. Aerosp. Sci., 41(1):1-28, 2005. ISSN 03760421. doi: https://doi.org/10.1016/j.paerosci.2005.02.001. URL http://www.sciencedirect.com/science/article/pii/S0376042105000102

[13] G. Sun and S. Wang. A review of the artificial neural network surrogate modeling in aerodynamic design. J. Aerospace Eng., 233(16):5863-5872, 2019. doi: 10.1177/0954410019864485. URL https://doi.org/10.1177/0954410019864485.

[14] S. Bhatnagar, Y. Afshar, S. Pan, K. Duraisamy, and S. Kaushik. Prediction of aerodynamic flow fields using convolutional neural networks. Computational Mechanics, 64(2):525-545, Jun 2019. ISSN 1432-0924. doi: 10.1007/s00466-019-01740-0. URL http://dx.doi.org/10.1007/s00466-019-01740-0.

[15] J. Chen, J. Viquerat, and E. Hachem. U-net architectures for fast prediction of incompressible laminar flows, 2019.

[16] F. de Avila Belbute-Peres, T. Economon, and Z. Kolter. Combining differentiable PDE solvers and graph neural networks for fluid flow prediction. In Proceedings of Machine Learning and Systems 2020, pages 11167-11176, 2020.

[17] K. Um, R. Brand, P. Holl, R. Fei, and N. Thuerey. Solver-in-the-loop: Learning from differentiable physics to interact with iterative PDE-solvers. Advances in Neural Information Processing Systems, 2020.

[18] N. Thuerey, K. Weissenow, L. Prantl, and X. Hu. Deep learning methods for Reynolds-averaged Navier-Stokes simulations of airfoil flows, 2018.

[19] S. Eismann, S. Bartzsch, and S. Ermon. Shape optimization in laminar flow with a label-guided variational autoencoder, 2017.

[20] J. Viquerat and E. Hachem. A supervised neural network for drag prediction of arbitrary 2D shapes in low Reynolds number flows, 2019.

[21] J. Li, M. Zhang, J. R. R. A. Martins, and C. Shu. Efficient aerodynamic shape optimization with deep-learning-based geometric filtering. AIAA J., 58(10):4243-4259, 2020. doi: 10.2514/1.J059254. URL https://doi.org/10.2514/1.J059254. 
[22] S. A. Renganathan, R. Maulik, , and J. Ahuja. Enhanced data efficiency using deep neural networks and gaussian processes for aerodynamic design optimization, 2020.

[23] V. Michelassi, L. Chen, R. Pichler, and R. D. Sandberg. Compressible direct numerical simulation of low-pressure turbines-Part II: effect of inflow disturbances. ASME. J. Turbomach., 137:071005, 2015.

[24] F. de Avila Belbute-Peres, K. Smith, K. Allen, J. Tenenbaum, and J. Z. Kolter. End-to-end differentiable physics for learning and control. In $A d$ vances in neural information processing systems, 2018.

[25] P. Holl, N. Thuerey, and V. Koltun. Learning to control PDEs with differentiable physics. In International Conference on Learning Representations, 2019 .

[26] O. Pironneau. On optimum profiles in Stokes flow. J. Fluid Mech., 59: 117-128, 1973.

[27] O. Pironneau. On optimum design in fluid mechanics. J. Fluid Mech., 64: 97-110, 1974.

[28] R. Glowinski and O. Pironneau. On the numerical computation of the minimum-drag profile in laminar flow. J. Fluid Mech., 72:385-389, 1975.

[29] R. Glowinski and O. Pironneau. Towards the computation of minimum drag profiles in viscous laminar flow. Appl. Math. Modelling, 1:58-66, 1976.

[30] D. W. Kim and M.-U. Kim. Minimum drag shape in two-dimensional viscous flow. Int. J. Numer. Methods Fluids, 21(2):93-111, 2005. doi: $10.1080 / 10618560410001710469$.

[31] E. Katamine, H. Azegami, T. Tsubata, and S. Itoh. Solution to shape optimisation problems of viscous fields. Int. J. Comut. Fluid Dyn., 19(1): 45-51, 2005. doi: 10.1080/10618560410001710469.

[32] T. Kondoh, T. Matsumori, and A. Kawamoto. Drag minimization and lift maximization in laminar flows via topology optimization employing simple objective function expressions based on body force integration. Struct. Multidiscipl. Optim., 45:693-701, 2012.

[33] R. Yondo, E. Andrés, and E. Valero. A review on design of experiments and surrogate models in aircraft real-time and many-query aerodynamic analyses. Prog. Aerosp. Sci., 96:23-61, 2018. ISSN 0376-0421. doi: https://doi.org/10.1016/j.paerosci.2017.11.003. URL http://www.sciencedirect.com/science/article/pii/S0376042117300611.

[34] A. Paszke, S. Gross, F. Massa, A. Lerer, et al. Pytorch: An imperative style, high-performance deep learning library. In Advances in neural information processing systems, pages 8026-8037, 2019. 
[35] D. Kraft. Self-consistent gradient flow for shape optimization. Optim. Methods Softw., 32(4):790-812, 2017. doi: 10.1080/10556788.2016.1171864. URL https://doi.org/10.1080/10556788.2016.1171864. PMID: 28670104.

[36] S. V. Patankar and D. B. Spalding. A calculation procedure for heat, mass and momentum transfer in three-dimensional parabolic flows. In Numerical Prediction of Flow, Heat Transfer, Turbulence and Combustion, pages 5473. Elsevier, 1983.

[37] H. K. Versteeg and W. Malalasekera. An Introduction to Computational Fluid Dynamics, chapter 6. Pearson Education Limited, Essex CM20 2JE England, 2 edition, 2007.

[38] T. D. Economon, F. Palacios, S. R. Copeland, T. W. Lukaczyk, and J. J. Alonso. SU2: an open-source suite for multiphysics simulation and design. AIAA J., 54(3):828-846, 2016. doi: 10.2514/1.J053813. URL https://doi.org/10.2514/1.J053813.

[39] S. C. Dennis and G. Chang. Numerical solutions for steady flow past a circular cylinder at Reynolds numbers up to 100. J. Fluid Mech., 42:471489, 1970 .

[40] D. J. Tritton. Experiments on the flow past a circular cylinder at low Reynolds numbers. J. Fluid Mech., 6(4):547-567, 1959. doi: 10.1017/ S0022112059000829.

[41] S. Osher and J. A. Sethian. Fronts propagating with curvature dependent speed: algorithms based on Hamilton-Jacobi formulations. J. Comput. Phys., 79:12-49, 1988.

[42] J. A. Sethian. Computational Methods for Fluid Flow, chapter 16, 17. Cambridge University Press, Cambridge CB2 2RU, UK, 2 edition, 1999.

[43] J. A. Sethian and P. Smereka. Level set methods for fluid interface. Annu. Rev. Fluid Mech., 35:341-372, 2003. doi: 10.1146/annurev.fluid.35.101101. 161105 .

[44] A. Baeza, C. Castro, F. Palacios, and Zuazua E. 2D Navier-Stokes shape design using a level set method. AIAA Paper 2008-172, 2008. doi: 10. 2514/6.2008-172.

[45] S. Zahedi and A.-K. Tornberg. Delta function approximations in level set methods by distance function extension. J. Comp. Phys., 229:2199-2219, 2010. doi: $10.1016 / j . j c p .2009 .11 .030$.

[46] J. A. Sethian. Fast marching methods. SIAM Rev., 41:199-235, 1999. doi: $10.1137 /$ S0036144598347059.

[47] L. He, C.-Y. Kao, and S. Osher. Incorporating topological derivatives into shape derivatives based level set methods. Comput. Fluids, 225:891-909, 2007. doi: $10.1016 /$ j.jcp.2007.01.003. 
[48] B. A. Gardner and M. S. Selig. Airfoil design using a genetic algorithm and an inverse method. AIAA Paper 2003-0043, 2003.

[49] F. Yang, Z. Yue, L. Li, and W. Yang. A new curvature-controlled stacking-line method for optimization design of compressor cascade considering surface smoothness. J. Aerospace Eng., 232:459-471, 2018. doi: $10.1177 / 0954410016679433$.

[50] X. Zhang, X. Qiang, J. Teng, and W. Yu. A new curvature-controlled stacking-line method for optimization design of compressor cascade considering surface smoothness. J. Aerospace Eng., 234:1061-1074, 2020. doi: $10.1177 / 0954410019894119$.

[51] O. Ronneberger, P. Fischer, and T. Brox. U-net: Convolutional networks for biomedical image segmentation. In N. Navab, J. Hornegger, W. M. Wells, and A. F. Frangi, editors, Medical Image Computing and Computer-Assisted Intervention - MICCAI 2015, pages 234-241, Cham, 2015. Springer International Publishing. ISBN 978-3-319-24574-4.

[52] D. P. Kingma and J. Ba. Adam: A method for stochastic optimization, 2014.

[53] J. Ling, A. Kurzawski, and J. Templeton. Reynolds averaged turbulence modelling using deep neural networks with embedded invariance. J. Fluid Mech., 807:155-166, 2016.

[54] S. Sen, S. Mittal, and G. Biswas. Steady separated flow past a circular cylinder at low Reynolds numbers. J. Fluid Mech., 620:89-119, 2009. doi: $10.1017 /$ S0022112008004904. 